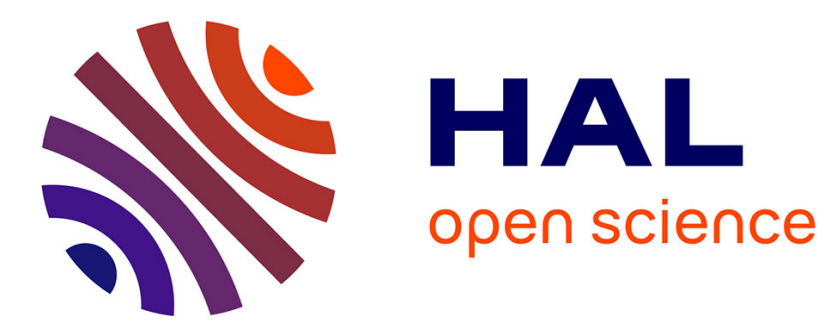

\title{
GRAPH SMALL CANCELLATION THEORY APPLIED TO ALTERNATING LINK GROUPS
}

\author{
Rémi Cunéo, Hamish Short
}

\section{To cite this version:}

Rémi Cunéo, Hamish Short. GRAPH SMALL CANCELLATION THEORY APPLIED TO ALTERNATING LINK GROUPS. Journal of Knot Theory and Its Ramifications, 2012, 21 (11), pp.1250113. 10.1142/S0218216512501131. hal-01263143

\section{HAL Id: hal-01263143 https://hal.science/hal-01263143}

Submitted on 4 Feb 2016

HAL is a multi-disciplinary open access archive for the deposit and dissemination of scientific research documents, whether they are published or not. The documents may come from teaching and research institutions in France or abroad, or from public or private research centers.
L'archive ouverte pluridisciplinaire HAL, est destinée au dépôt et à la diffusion de documents scientifiques de niveau recherche, publiés ou non, émanant des établissements d'enseignement et de recherche français ou étrangers, des laboratoires publics ou privés. 


\title{
Graph small cancellation theory applied to alternating link groups
}

\author{
Rémi Cunéo and Hamish Short
}

December 13, 2011

\begin{abstract}
We show that the Wirtinger presentation of a prime alternating link group satisfies a generalized small cancellation theory condition. This gives a simplification of Weinbaum's solution to the word and conjugacy problems for these groups.
\end{abstract}

\section{Introduction}

To show that an alternating prime link group has a solvable word and conjugacy problem, C.M.Weinbaum ([9], see also [4]) uses that the Dehn presentation of this group almost verifies the small cancellation conditions $C(4)-T(4)$. In fact Weinbaum works with a presentation of a free product of the link group and an infinite cyclic group, which implies some technical detours in the proof. In [1], it is shown that the Dehn presentation corresponds to a $C A T(0)$ complex.

The more common Wirtinger presentation does not verify these classical small cancellation conditions, but we shall show here that it does satisfy the conditions of a recent version of new small cancellation theory, due to Gromov (see [3]) and Ollivier (see [6]) using graphs. The usual small cancellation theory methods then give solutions to the word and conjugacy problems.

First, we define the presentation associated to a finite, labelled, oriented and reduced graph $\Gamma$, such that generators are the labels and relators are the words read on a generating family of cycles of $\Gamma$.

Given a minimal Van Kampen diagram $D$, we then subdivide this diagram into megatiles (essentially, a megatile is a maximal subdiagram of $D$, implicitly used in [6]) and consider a small cancellation type condition on this megatile diagram.

An important difference with the classical small cancellation theory from [4] is that a piece is a word which has two distinct immersions in $\Gamma$.

Under usual small cancellation conditions (from the graph viewpoint), the megatiles are simply connected, so the megatile diagram $D^{\prime}$ is a diagram, considering each megatile as a face. 
The first important result, shown in [6], is that each megatile satisfies an linear isoperimetric inequality.

We give a correspondance between the two viewpoints: if $\Gamma$ satisfies the non metric conditions $C(p)-T(q)_{O}$ ("O" for "Ollivier"), then each megatile diagram of a minimal diagram is a $[p, q]$-map, in the terminology of [4]. Then we obtain an analogous result to the main technical result of classical small cancellation theory in the non-metric case (see [4] theorem 6.3 p 262 and theorem $7.6 \mathrm{p} \mathrm{265)}$ giving solutions to the word and conjugacy problems, from the graph viewpoint: see theorem 5 .

As an application of this technical theorem, we have the following theorem and its immediate corollary:

Theorem 6. Let $\Gamma$ be the dual graph associated to a regular prime alternating link projection $P$, let $\mathcal{R}$ be the natural "visual" basis of circuits of length 4 . Then:

1. The presentation associated to the graph $(\Gamma, \mathcal{R})$ is the Wirtinger presentation of the link group.

2. $(\Gamma, \mathcal{R})$ satisfies the small cancellation conditions $C(4)-T(4)_{O}$.

Together the above two theorems immediately give:

Corollary 7 (Weinbaum [9]). Groups of prime alternating links have solvable word and conjugacy problems.

It is also clear that if a prime alternating presentation $\mathcal{P}(\Gamma, \mathcal{R})$ of a knot contains at least one crossing, then the knot is not trivial, as it is easy to show that the shortest relation has length 4 , and all the generators of $\mathcal{P}(\Gamma, \mathcal{R})$ are meridians or their inverses (see [5]). If the group were $\mathbb{Z}$, then there would be a relation of length 2 .

With a little more work, one can show that prime alternating link presentations with at least one crossing correspond to non-trivial link.

\section{Context}

We summarize here some basic definitions and properties of this new viewpoint: for more details, see [2].

Throughout, $\Gamma$ will be a finite, oriented, not necessarily connected graph, without vertices of degree $1 . \Gamma$ is labelled by letters, which form a set denoted by $S$. In addition, we shall suppose that $\Gamma$ is reduced (two consecutives edges 
of the graph are never respectively labelled by a generator and its inverse) and there are no redundant circuits.

We define the finitely presented group $G$, associated with $\Gamma$ as follows: $S$ is the set of the generators. A set of relators, $\mathcal{R}$, is a set of words read on a basis (or generating family) of cycles (circles immersed in $\Gamma$ ) or circuits (circles embedded in $\Gamma$ ) of $\Gamma$, which generate $H_{1}(\Gamma)$. We shall assume that $\mathcal{R}$ always contains the shortest circuit, of length $\gamma(\gamma$ is the girth of $\Gamma)$.

This gives a finite presentation $\mathcal{P}(\Gamma, \mathcal{R})=\langle S \mid \mathcal{R}\rangle$ of the group $G=$ $\frac{F(S)}{\langle\langle\mathcal{R}\rangle\rangle}=\langle S \mid \mathcal{R}\rangle$, where $\langle\langle\mathcal{R}\rangle\rangle$ is the normal subgroup of $F(S)$ generated by $\mathcal{R}$. Notice that $\langle\langle\mathcal{R}\rangle\rangle$ is independent of the choice of the set $\mathcal{R}$.

Classical small cancellation theory uses conditions on the lengths of pieces relative to the lengths of relators, where a piece is a common subword of two relators. A simple example illustrates the difference between the two approaches. In classical small cancellation theory (see[4]), in the presentation $\mathcal{P}=\left\langle a, b, c \mid b a^{-1}, b c^{-1}\right\rangle, b$ is a piece (common initial prefix of two distinct relators) of length 1 for the word metric, and is half the length of the relators containing it.

In this "graph small cancellation theory", a piece is defined as follows:

Definition 1. Let $\Gamma$ be a finite labelled oriented graph. A piece is a word labelling two distinct path immersions in $\Gamma$.

In the example, consider the $\theta$-curve $\Gamma$ consisting of two vertices joined by three edges labelled by $a, b$ and $c$. As each of $a, b$ and $c$ has an unique immersion in $\Gamma$, there is no piece in $\Gamma$. It is clear how to choose $\mathcal{R}$ such that $\mathcal{P}(\Gamma, \mathcal{R})=\mathcal{P}=\left\langle a, b, c \mid b a^{-1}, b c^{-1}\right\rangle$.

Remark 2. It is hard to take into account torsion in this version of the theory: if there is an embedded loop labelled by a proper power $w^{n}$, then it is itself a piece. For more on this see [2].

A (van Kampen) diagram over a finite presentation $\mathcal{P}=\langle S \mid \mathcal{R}\rangle$ is a finite, planar, connected, simply connected 2-complex $D$, with oriented edges labelled in $S$, such that the boundary of each face is labelled by a word of $\mathcal{R}$ (up to cyclic permutation and inversion). The label on the outer boundary (the boundary of the complement of $D$ in $\mathbb{R}^{2}$ ) is a word $w$ in the free semigroup on $S \cup S^{-1}$. We also say that $D$ is a diagram for $w$ over $\mathcal{P}$.

A diagram for $w$ is minimal if all other diagrams for $w$ have at least as many faces. Note that minimal diagrams are reduced. Van Kampen's 
original interest in these diagrams is the fact (see [8]) that every word $w$ in $\langle\langle\mathcal{R}\rangle\rangle$ has a diagram over $\mathcal{P}$. We define the area of $w \in\langle\langle\mathcal{R}\rangle\rangle, \operatorname{Area}_{\mathcal{P}}(w)$, to be the number of 2 -cells in a minimal diagram for $w$ over $\mathcal{P}$. We say that the presentation satisfies a linear (quadratic, cubic, exponential, recursive) isopermetric inequality if there is a linear (quadratic, cubic, exponential, recursive) function $f_{\mathcal{P}}: \mathbb{N} \rightarrow \mathbb{R}$ such that $\operatorname{Area}(w) \leq f_{\mathcal{P}}(|w|)$. A standard result of this domain is that the word problem of a presentation is solvable if and only if it has a recusive isoperimetric inequality. As usual, $|w|$ denotes the length of the word $w$ (in the free group or semigroup $F(S)$, according to the context).

Let $D$ be a minimal diagram over a graph presentation $\mathcal{P}(\Gamma, \mathcal{R})$.

We say that two open faces of $D$ are $\Gamma$-adjacent if their boundaries in $D$ contain a common edge originating from $\Gamma$ (that is, the two lifts of the common edge is at the same place in $\Gamma$ ). We say that a vertex of $D$ originates from $\Gamma$ if it only belongs to closed edges coming from $\Gamma$.

The reflexive and transitive closure of the $\Gamma$-adjacency relation is an equivalence relation, and a megatile $M$ is formed from the union of all elements of an equivalence class, together with all open edges originating from $\Gamma$ between $\Gamma$-adjacent faces of this class and vertices which belong only to boundaries of these open edges.

Seen as an abstract complex, $M$ has a boundary, denoted $\partial M$, which is a not necessarily connected 1-complex, and $\partial M$ lifts to $\Gamma$. Notice that edges of $\partial M$ do not originate from $\Gamma$, and distinct edges in $\partial M$ may be identified in $D$ (see figure 1), that is $M \cup \partial M$ is not necessarily homeomorphic to the closure of $M$ in $D$. In figure 1, the lower-left megatile $M$ of $D$ is a disc, with boundary $\partial M$ a circle, but the closure of $M$ in $D$ is an annulus.

This notion of megatile is implicitly used in [6] (where faces are called "tiles"). Notice that a face of a minimal diagram belongs to one and only one megatile, and that a megatile of a minimal diagram $D$ is not necessarily simply connected (see the figure 1). But under the usual small cancellation conditions (e.g. $C(4)-T(4)$, see theorem 5.1), each megatile of a minimal diagram is simply connected (two megatiles of the figure 1 are impossible under these conditions).

The megatile diagram of $D$, denoted by $D^{\prime}$, is the 2-complex, unique by construction, built from $D$, by deleting open edges of $D$ originating from $\Gamma$ and vertices of $D$ originating from $\Gamma$ (see the figure 1 ).

In a diagram $D$, we can distinguish a $D$-edge, which is an edge of $D$ 


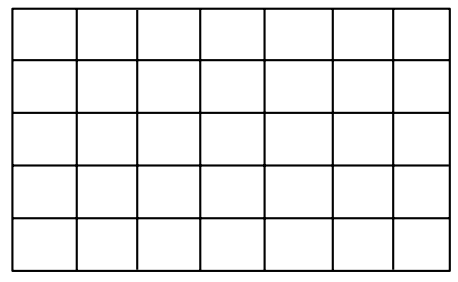

$D$

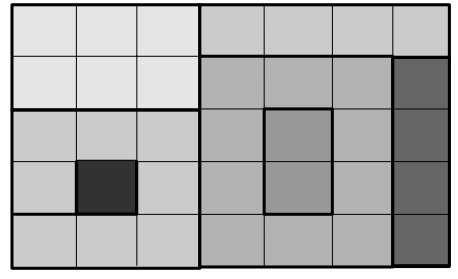

Partition of $D$ in megatiles (grey regions)

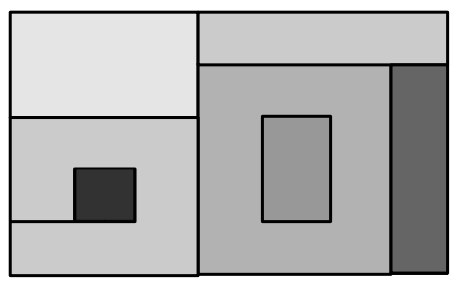

Megatile diagram of $D$

Figure 1: Megatile diagram of a diagram $D$

labelled by a generator or its inverse and a $D^{\prime}$-edge, which is an arc of the megatile diagram $D^{\prime}$ of $D$, labelled by a word, such that the extremal vertices have degree at least 3 and the others have degree 2 (in $D^{\prime}$ ).

We repeat that an important property of each megatile $M$ is that every loop in $\partial M$ lifts to a cycle in $\Gamma$.

\section{Results in graph small cancellation theory}

We give now two important lemmas about megatiles; the first one comes from [6]:

Lemma 3. Let $\Gamma$ be a graph, $\mathcal{R}$ a basis of $\Gamma$, of girth $\gamma$ and diameter $\Delta$. Let

$$
\mu=\max \{\operatorname{Area}(w)|| w \mid \leq 3 \Delta, w \text { labels a cycle in } \Gamma\}
$$

Let $D$ be a minimal diagram over $(\Gamma, \mathcal{R})$ and $M$ a simply connected megatile of $D$.

Then:

- $M$ satisfies a linear isoperimetric inequality.

More precisely, $M$ contains at most $\frac{3 \mu}{\gamma}|\partial M|$ faces. 
- If $M$ and $M^{\prime}$ are two megatiles of $D$, each arc of $\partial M \cap \partial M^{\prime}$ is labelled by a piece (not necessarily maximal, i.e. included in a longer piece of $\Gamma$ ).

We say that a graph $\Gamma$ verifies the $C(p)_{O}$ condition, $p \geq 2$, when no cycle of $\Gamma$ can be decomposed into fewer than $p$ pieces (with disjoint interior).

Let $q \geq 3$. We say that $\Gamma$ verifies the $T(q)_{O}$ condition when:

If there are $h(3 \leq h<q)$ paths (of length 2) in $\Gamma$ labelled $p_{1} p_{2}^{-1}$, $p_{2} p_{3}^{-1}, \ldots, p_{h} p_{1}^{-1}$, with $p_{1}, p_{2}, \ldots, p_{h}$ generators (or their inverses, and $p_{i} \neq$ $p_{i+1}(\bmod h)$ as $\Gamma$ reduced $)$, which lift to $\Gamma$, then the $h$ paths have the same vertex of $\Gamma$ as their midpoint.

These last technical definitions imply more simple and convenient properties:

Properties 4. Let $D$ be a minimal diagram over $(\Gamma, \mathcal{R})$, and $D^{\prime}$ the megatile diagram of $D$.

Then:

1. If $\Gamma$ satisfies $C(p)_{O}$, the boundary of each internal simply connected megatile $M$ of $D^{\prime}$ (internal means that $\partial D^{\prime} \cap \partial M$ contains no edge) is composed of at least $p D^{\prime}$-edges. (One says that $D^{\prime}$ satisfies $C(p)$ )

2. If $\Gamma$ satisfies $T(q)_{O}$, the degree of each internal vertex of $D^{\prime}$ is at least $q$. (One says that $D^{\prime}$ satisfies $T(q)$ )

The proof is essentially the same than in [4] (lemma $2.2 \mathrm{p} \mathrm{242).}$

Theorem 5. Suppose $(\Gamma, \mathcal{R})$, of girth $\gamma$, satifies $C(p)-T(q)_{O}$ for $(p, q) \in$ $\{(4,4),(6,3),(3,6)\}$. Let $D$ be a minimal diagram over $\Gamma$ and $D^{\prime}$ the megatile diagram of $D$. Then:

1. Each megatile of $D$ is simply connected. Hence, one can consider $D^{\prime}$ as a diagram, whose faces are megatiles of $D$.

2. The group $G$ associated to $\Gamma$ is torsion free.

3. The word and conjugacy problems are solvable for $G$ (there is a cubic isoperimetric inequality). 
Sketch of the proof:

One first uses the $C(p)-T(q)_{O}$ conditions to show (1). The megatile diagram $D^{\prime}$ of every minimal diagram $D$ then satisfies the $C(p)-T(q)$ conditions, as said in the terminology of $[4], D^{\prime}$ is a $[p, q]$-map. Then, using standard methods and results of classical small cancellation theory, it is not hard to prove:

2. The fact that there are no $[p, q]$-maps on a sphere implies there is no torsion.

3. A $[p, q]$-map satisfies at least a quadratic isoperimetric inequality. Using lemma 3 in each megatile of $D^{\prime}$, we obtain a cubic isoperimetric inequality. It is easy to show that the lengths of the boundaries of the megatiles are bounded by a linear function of the boundary of $D$ (see [2], theorem I.45 p $66)$.

\section{Application to prime alternating link groups \\ a. Construction of a link graph}

One considers an oriented link $L$ embedded in $\mathbb{S}^{3}$ and a regular projection $P(L)$ of this link. At each double point of this projection, two opposite germs of the arcs are identified to signify the presence of an overcrossing and an undercrossing. By abuse of language, in this projection, arcs stop to each undercrossing. As in [4] condition (1) p 268, we can suppose that there are four distinct regions at each crossing.

One orients and labels each arc (of each component) between two crossings of this projection (in the same sense) by a generator; these generators correspond to those of the Wirtinger presentation; one obtains one relator of length 4 per crossing. This gives a presentation of the fundamental group of the link complement (see [7] pp 56-59).

The graph $\Gamma(L)$ (or simply $\Gamma$ ) of the link is obtained by duality from the regular projection. To each open and connected region of the regular projection, we associate a vertex of the graph and to each arc of the regular projection an edge of the graph which is transverse to this arc.

Then, one agrees on a rule for the labelling and the orienting of the graph:

If one imagines the link projected onto the graph, seen from above, and if one follows each component of the link in the positive sense, one labels each edge of the graph by the same generator which labels the transverse arc of the link, oriented, by convention, from left to right. 
At each double point of the regular projection (a double point corresponds to an overcrossing of the link projection), there is in general a circuit of length 4 in $\Gamma$, where one generator conjugates another to a third. This is a Wirtinger relator (see [7] p 56-59).

An example of this construction is given by the figure 2 .
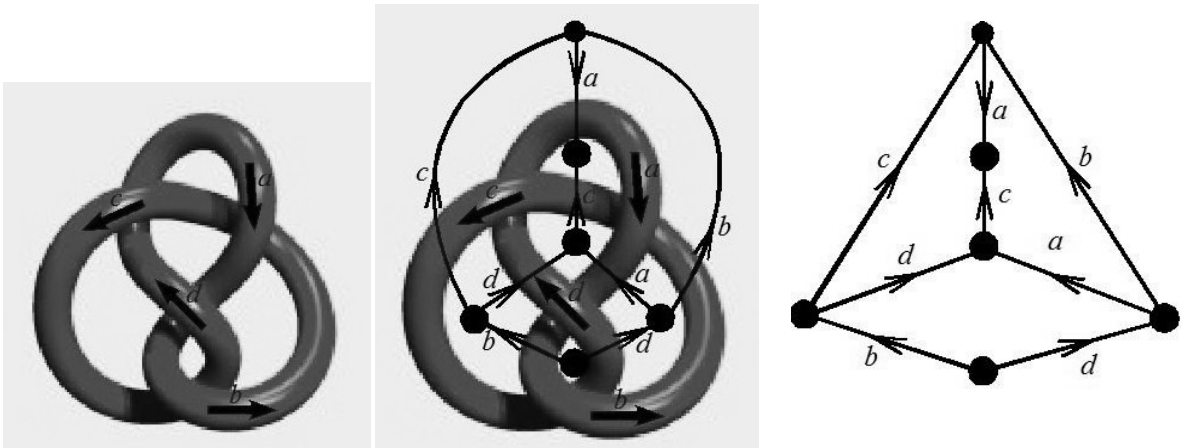

Figure 2: Graph associated to (the regular projection of) the figure eight knot giving $\mathcal{P}(\Gamma)=\left\langle a, b, c, d \mid b c b^{-1} d^{-1}, c a c^{-1} d^{-1}, d a d^{-1} b^{-1}\right\rangle$. See [7] p 58 where $x_{1}=b, x_{2}=a, x_{3}=d, x_{4}=c$.

\section{b. Word and conjugacy problems}

Theorem 6. Let $\Gamma$ be the graph associated to a regular prime alternating link projection $P$ as above.

Let $\mathcal{R}$ be the natural "visual" basis of circuits of length bounding compact regions of the complement of $P$ in $\mathbb{R}^{2}$.

Then:

1. The presentation associated to the graph $(\Gamma, \mathcal{R})$ is the Wirtinger presentation of the link group.

2. $(\Gamma, \mathcal{R})$ satisfies the small cancellation conditions $C(4)-T(4)_{O}$.

Corollary 7. Groups of prime alternating links have solvable word and conjugacy problems.

Proof

We show that the graph of a prime alternating link (in particular the figure eight knot) satisfies the $C(4)-T(4)_{O}$. 
Each generator occurs exactly twice in a single circuit of length 4 of the graph of a prime alternating link, since the arc of the projection of the link labelled by this generator, meets the boundary of exactly 4 regions of the complement of the projection (corresponding to four vertices of the graph, two couples of which form an edge labelled by the generator involved).

More precisely, as we can see in the figure 3, these two immersions of an edge labelled " $a$ " in the graph $\Gamma$, in the same cycle of length 4 , are separated by two distinct generators and have opposite orientation (in this cycle).

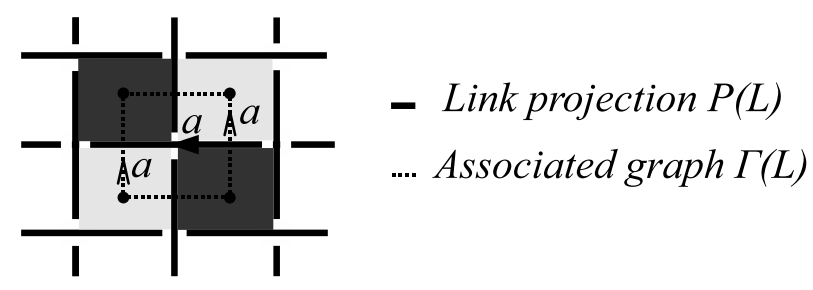

Figure 3: Each generator occurs exactly twice in the link graph (and once in each of two other circuits of length 4), in the same cycle of length 4, "alternately" and with opposite orientation.

We see easily that the only possible pieces are of length 1 . Indeed, the only way to have a piece of length 2 in the graph is shown in figure 4 and this implies that the link is not alternating.



Figure 4: Piece of length 2 in the graph

For example, in the graph of the figure eight knot, one can read the words $a^{-1} b$ (or $b^{-1} a$ ) and $b a$ (or $a^{-1} b^{-1}$ ) in the graph, but the words $a^{ \pm 1} b^{ \pm 1}$ do not appear elsewhere in the graph.

$C(4)_{O}$ condition:

In the example of the figure eight knot, it is clear that all the cycles of $\Gamma$ are of length at least 4 . As each piece has length 1 and each generator is a piece, the condition $C(4)_{O}$ is therefore satisfied. 
In general, the regular projection of a link is a bipartite graph (this is a topological checkerboard). Therefore, by construction of the link graph, the cycles of the graph have even length (one cycle passes alternately a black region, a white region, and returns to its starting point).

If a cycle of length 2 comes from a link projection, then the two edges of the graph, joining two vertices of the graph would form an embedded $\mathbb{S}^{1}$ in the plane, meeting the link projection in two points; which is characteristic of a non prime link, as the projection is regular (see [4] condition (2) and figure 5).

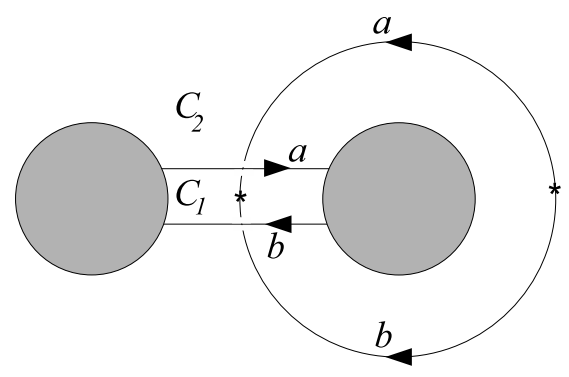

Figure 5: Origin of a cycle of length 2

Pieces have length 1, each generator is a piece and cycles have length at least 4 , so the $C(4)_{O}$ condition is verified.

$T(4)_{O}$ condition:

With the convention erasing vertices of degree 2 (see [4] p 242), we just have to check that in a minimal diagram $D$ over $\Gamma$, every internal vertex of degree 3 is internal to a megatile of $D$.

In the example of the figure eight knot, we just list all possible configurations of vertices of degree 3 to realise that this is the case.

Now, we argue by contradiction. Let $v$ be a vertex of degree 3 , internal to $M$, the subdiagram of $D$ composed of three faces $F_{a b}, F_{b c}$ and $F_{a c}$ of $\Gamma$ $\left(\{v\}=\partial F_{a b} \cap \partial F_{b c} \cap \partial F_{a c}\right)$. We use the notations of figure 6 .

Case $\mathrm{n}^{\circ} 1$ : at least one of the three edges $a, b, c$ at $v$ originates from $\Gamma$.

Without loss of generality, suppose the edge is labelled $b$, then $\left(F_{a b}, F_{b c}\right)$ is (or is included in) a megatile of $D$ and the tripod $(a ; b ; c)$ of vertex $v$ lifts entirely to $\Gamma$ (with the vertex $v$ ). As $a c^{-1}$, of length 2 , is not a piece, the 


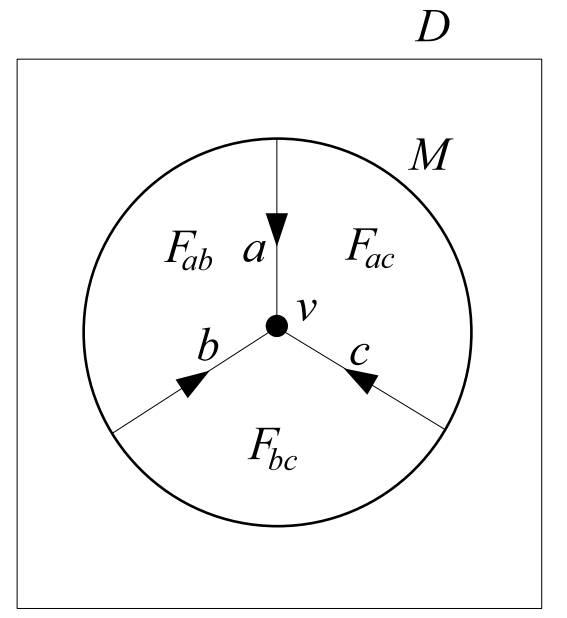

Step 1 : lifts of a

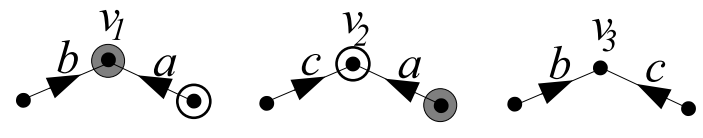

Step 2 : lifts of $b$

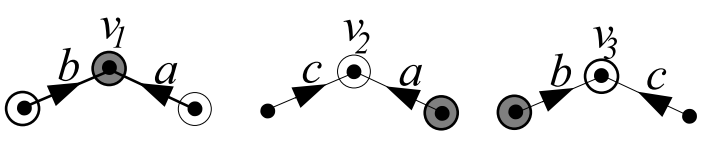

Step 3 : lifts of $c$

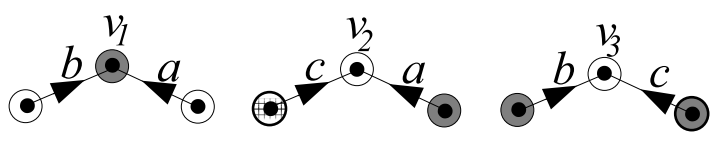

Impossible

Figure 6: Vertex of degree 3 in a minimal diagram

boundary of the face $F_{a c}$ lifts together with the unique lift of the path labelled by this word in $\Gamma$.

It follows in this case that $M$ is contained in a megatile of $D$, the three edges originate from $\Gamma$ and $v$ is an internal vertex of a megatile of $D$.

Case $\mathrm{n}^{\circ} 2$ : none of the edges $a, b, c$ originates from $\Gamma$.

So each of the three letters $a, b, c$ has exactly two distinct lifts in $\Gamma$.

As the pieces of $\Gamma$ are of length 1 , there cannot be two distinct lifts of the paths labelled by $a b^{-1}, b c^{-1}, c a^{-1}$ (or their inverses), so $v$ lifts to $\Gamma$ as an internal vertex of each of these three paths.

But $a$ (for example) appears exactly twice in $\Gamma$ in a circuit of length 4 , with opposite orientations, separated by two different generators (neither being $a$, see figure 3 ).

We use a colouring argument (see figure 6):

One colours vertices of $\Gamma$ alternately in black and white (from the checkerboard colouring of the projection). According to the previous remark, the two initial points (similarly, the two terminal points) of the edge labelled $a$ have different colours in $\Gamma$. Likewise for $b$ and $c$.

The vertex $v$ (of the figure 6) has three distinct lifts $v_{1}, v_{2}$ and $v_{3}$ in $\Gamma$. As the edge of $D$ labelled by $a$ is on the boundary of two megatiles of $D$, it 
lifts necessarily to two different places in $\Gamma$. By doing the same with $b$, then with $c$, we get a contradiction on colorations: we have the same coloring for both initial points of the two distinct edges of the graph labelled by $c$. This is impossible. So every internal vertex of degree 3 is included in a megatile. The $T(4)_{O}$ condition is satisfied for the graph.

\section{References}

[1] M.R. Bridson, A. Haefliger, 'Metric spaces of non-positive curvature', Springer-Verlag (1999).

[2] R. Cunéo, Généralisation d'une méthode de petites simplifications due à Mikhaïl Gromov et Yann Ollivier en géométrie des groupes, thesis, Université de Provence, 2011.

[3] M. Gromov, Random Walk in Random Groups, GAFA, Geom. Funct. Anal. 13 (2003), nº, $73-146$.

[4] R.C. Lyndon, P.E. Schupp, 'Combinatorial Group Theory', Springer (1977).

[5] I. Moffatt, A new proof that alternating links are non-trivial, Journal of Knot theory and its Ramifications, vol. 19 (2010), number 6, 821-828.

[6] Y. Ollivier, On a small cancellation theorem of Gromov, Bull. Belg. Math. Soc. Simon Stevin, 13, Number 1 (2006), 75-89.

[7] D. Rolfsen, 'Knots and Links', Publish or Perish (1976).

[8] E.R. van Kampen, On some lemmas in the theory of groups, American Journal of Mathematics, number 55 (1933), 261-267.

[9] C.M. Weinbaum, The word and conjugacy problem for the knot group of any tame prime alternating knot., Proc. A.M.S. 22, 22-26 (1971).

Remi.Cuneo@free.fr

Hamish.Short@latp.univ-mrs.fr

L.A.T.P. UMR 6632, Centre Mathématiques et Informatique, 37 Rue Joliot Curie, 13453 Marseille, France 